\section{About the Materials} Research Society

The Materials Research Society (MRS), a not-forprofit scientific association founded in 1973, promotes interdisciplinary goal-oriented basic research on materials of technological importance. Membership in the Society includes over 14,000 scientists, engineers, and research managers from industrial, government, and university research laboratories in the United States and close to 70 countries.

The Society's interdisciplinary approach differs from that of single-discipline professional societies because it promotes information exchange across the many technical fields touching materials development. MRS sponsors two major internationa annual meetings encompassing approximately 70 topical symposia, and also sponsors numerous single-topic scientific meetings. The Society recognizes professional and technical excellence and fosters technical interaction in local geographic regions through Sections and University Chapters.

MRS participates in the international arena of materials research through the International Union of Materials Research Societies (IUMRS). MRS is a member of ASTRA and is an affiliate of the American Institute of Physics.

MRS publishes symposium proceedings, MRS Bulletin, Journal of Materials Research, and other publications related to current research activities.

MRS Bulletin (ISSN: 0883-7694) is published 12 times a year by the Materials Research Society, 506 Keystone Drive, Warrendale, PA 15086-7573. Copyright (02007, Materials Research Society. Permission required to reproduce content. Periodical postage paid at Warrendale, PA, and at additional mailing offices. POSTMASTER: Send address changes to MRS Bulletin in care of the Materials Research Society, at the address listed; tel. 724-7793003; fax 724-779-8313. Printed in the U.S.A.

Membership in MRS is \$105 annually for regular 30 for students. Dues include an allocetion of $\$ 29$ (\$17 for students) to a subscription to MRS Bulletin. Individual member subscriptions are for personal use only. Non-member subscription rates are $\$ 245$ for one calendar year (12 issues) within the U.S.A. and \$305 elsewhere. Single copies may be purchased for $\$ 40$ each. Requests from subscribers for missing journal issues will be honored without charge only if received within six months of the issue's actual date of publication; otherwise, the issue may be purchased at the single-copy price. MRS Bulletin is included in Current Contents ${ }^{\otimes}$ Engineering, Computing, and Technology; Current Contents ${ }^{\circledast} /$ Physical, Chemical, and Earth Sciences, the SciSearch ${ }^{\circledR}$ online database, Research Alert ${ }^{\boxplus}$, Science Citation Index and the Materials Science Citation

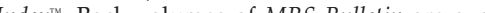
able on microfiche through University Microfilms Inc., 300 North Zeeb Road, Ann Arbor, M 48106, USA.

Send Letters to the Editor to: MRS Bulletin

Materials Research Society 506 Keystone Drive

Warrendale, PA 15086-7573 USA Fax 724-779-8313 Bulletin@mrs.org

Letters must include your name, affiliation, and full contact information.

\section{MIRSS BULLETIN}

Editorial Office • 506 Keystone Drive • Warrendale, PA 15086-7573 USA Tel. 724-779-3004 ext. 522; fax 724-779-8313; Bulletin@mrs.org; http://www.mrs.org/
Editor

Managing Editor

J. Meiksin

Technical Editor

A. Bertram Wilson

Editorial Assistant

B. Moriarty

Associate Technical Editor

T. Palucka

Design/Production

S. Franklin and D. Miller
E.L. Fleische
Advertising

M.E. Kaufold and

D.L. Watterson

Circulation

S. Forrest

Guest Editors

C.B. Arnold and A. Piqué

Special Contributors

J.J. Carvajal, E. Fitzgerald, K. Niece, and S. Trohalaki

\section{Special Consultants}

M.-I. Baraton, G.M. Chow, M. Mayo,

A. Nelmo Klein, and G. Solorzano
Book Review Board

J.H. Westbrook (Chair), C. Batich,

R.J. Matyi, L. Olafsen, S. Sant, and

J.-C. Zhao

Visiting Scientist

V.S. Arunachalam

Center for Study of Science,

Technology \& Policy

Bangalore, India

MRS Office of Public Affairs

499 South Capitol Street SW, Suite 600

Washington, DC 20003

\section{EDITORIAL BOARD}

P.S. Drzaic, Chair

Alien Technology Corp.

Morgan Hill, CA

V.S. Arunachalam

Center for Study of Science,

Technology \& Policy

Bangalore, India

I.W. Boyd

University College London

London, UK

R.C. Cammarata

Johns Hopkins University

Baltimore, MD

Laura Fornaro
University of Uruguay
Montevideo, Uruguay
A.L. Greer
University of Cambridge
Cambridge, UK
S.C. Moss
Aerospace Corporation
Los Angeles, CA
J.R. Weertman
Northwestern University
Evanston, IL

E. Werwa

Washington, DC

J.H. Westbrook

Brookline Technologies

Ballston Spa, NY

S.M. Yalisove

University of Michigan

Ann Arbor, MI

\title{
VOLUME ORGANIZERS
}

\section{6}

P.A. Midgley

UK

\section{7}

T.F. Kuech

University of

Wisconsin-Madison

2008

Y.-T. Cheng

Purdue University
C.A. Orme
Lawrence Livermore
National Laboratory

T.E. Mallouk

Pennsylvania State

University

R.S. Goldman

University of Michigan

\section{J.A. Rogers \\ University of Illinois at \\ Urbana-Champaign \\ J.-C. Zhao \\ Research Center}

\section{J.A. Nucci}

Max Planck Institute for

Metals Research

Germany

R. Krishnamoorti

University of Houston
R.A. Register

Princeton University

A. Sellinger

Institute of Materials

Research and Engineering

Singapore

\begin{tabular}{lll}
\hline \multicolumn{2}{c}{ 2007 MRS OFFICERS } \\
\hline President & Secretary & Immediate Past President \\
A.J. Hurd & D.S. Ginley & P.F. Green \\
Los Alamos National Laboratory & National Renewable Energy Laboratory & University of Michigan \\
& & \\
Vice President and President-Elect & Treasurer & Executive Director \\
C.A. Volkert & J.W.P. Hsu & Materials Research Society \\
Forschungszentrum Karlsruhe & Sandia National Laboratories & J.B. Ballance
\end{tabular}

\begin{tabular}{|c|c|c|c|c|c|c|c|c|}
\hline \multicolumn{9}{|c|}{ INTERNATIONAL UNION OF MATERIALS RESEARCH SOCIETIES } \\
\hline $\begin{array}{l}\text { President } \\
\text { G.M. Crean } \\
\text { Tyndall Natl. Inst. } \\
\text { Cork, Ireland }\end{array}$ & $\begin{array}{l}\text { First Vice President } \\
\text { H. Katz } \\
\text { Johns Hopkins University } \\
\text { Baltimore, MD, USA }\end{array}$ & \multicolumn{2}{|c|}{$\begin{array}{l}\text { Second Vice President } \\
\text { B.V.R. Chowdari } \\
\text { National University of Singapore } \\
\text { Singapore }\end{array}$} & $\begin{array}{l}\text { Secretary } \\
\text { J. Baglin } \\
\text { IBM Almaden Research Center } \\
\text { San Jose, CA, USA }\end{array}$ & \multicolumn{2}{|c|}{$\begin{array}{l}\text { Treasurer } \\
\text { H. Yamamoto } \\
\text { Nihon Univ. } \\
\text { Chiba, Japan }\end{array}$} & $\begin{array}{l}\text { Immediate Past President } \\
\text { L. Zhou } \\
\text { NIN } \\
\text { Xian, Shaanxi, China }\end{array}$ & $\begin{array}{l}\text { General Secretary } \\
\text { R.P.H. Chang } \\
\text { Northwestern University } \\
\text { Evanston, IL, USA }\end{array}$ \\
\hline \\
\hline $\begin{array}{l}\text { Australian Materia } \\
\text { J.S. Williams, Aus }\end{array}$ & $\begin{array}{l}\text { ls Research Society (A-MR } \\
\text { tralian National University }\end{array}$ & & $\begin{array}{l}\text { Materials Research } \\
\text { J. Ovejero García, } 0\end{array}$ & $\begin{array}{l}\text { Society of Argentina (MRS-Arge } \\
\text { IEA }\end{array}$ & & \multicolumn{3}{|c|}{$\begin{array}{l}\text { Materials Research Society of Singapore (MRS-S) } \\
\text { B.V.R. Chowdari, National University of Singapore }\end{array}$} \\
\hline $\begin{array}{l}\text { Brazil Materials R } \\
\text { E. Longo, UFSCar }\end{array}$ & esearch Society (B-MRS) & & $\begin{array}{l}\text { Materials Research } \\
\text { R.A. Mashelkar, Cou }\end{array}$ & $\begin{array}{l}\text { Ociety of India (MRS-I) } \\
\text { ncil of Scientific \& Industrial Res }\end{array}$ & & \multicolumn{3}{|c|}{$\begin{array}{l}\text { Materials Research Society of Taiwan (MRS-T) } \\
\text { J.-M. Liu, ITRI }\end{array}$} \\
\hline $\begin{array}{l}\text { Shinese Materials } \\
\text {. Zhou, NW Insti }\end{array}$ & $\begin{array}{l}\text { Research Society (C-MRS) } \\
\text { tute for Nonferrous Metal Re }\end{array}$ & Irch & $\begin{array}{l}\text { Materials Research } \\
\text { 0. Takai, Nagoya Un }\end{array}$ & $\begin{array}{l}\text { Society of Japan (MRS-J) } \\
\text { versity }\end{array}$ & & \multirow{3}{*}{\multicolumn{3}{|c|}{$\begin{array}{l}\text { Mexican Materials Research Society (Mexican-MRS) } \\
\text { P.H. Hernández Tejeda, Benemérita Universidad } \\
\text { Autónoma de Puebla }\end{array}$}} \\
\hline $\begin{array}{l}\text { European Material } \\
\text { J.P. Massue, E-N }\end{array}$ & $\begin{array}{l}\text { Is Research Society (E-MRS } \\
\text { IRS }\end{array}$ & & $\begin{array}{l}\text { Materials Research } \\
\text { J.-W. Park, Hanyang }\end{array}$ & $\begin{array}{l}\text { Society of Korea (MRS-K) } \\
\text { University }\end{array}$ & & & & \\
\hline \multicolumn{3}{|c|}{$\begin{array}{l}\text { Materials Research Society (MRS) } \\
\text { A.J. Hurd, Los Alamos National Laboratory }\end{array}$} & \multicolumn{3}{|c|}{$\begin{array}{l}\text { Materials Research Society of Russia (MRS-Russia) } \\
\text { N.Z. Lyakhov, Russian Academy of Sciences }\end{array}$} & & & \\
\hline
\end{tabular}

